\title{
Exon deletions and intragenic insertions are not rare in ataxia with oculomotor apraxia 2
}

\author{
Veronica Bernard ${ }^{1}$, Martina Minnerop ${ }^{2,3}$, Katrin Bürk ${ }^{4}$, Friedmar Kreuz ${ }^{5}$, \\ Gabriele Gillessen-Kaesbach ${ }^{1}$ and Christine Zühlke*1
}

\author{
Address: ${ }^{1}$ Institut für Humangenetik, Universität zu Lübeck, Lübeck, Germany, ${ }^{2}$ Klinik für Neurologie, Universität Bonn, Bonn, Germany, ${ }^{3}$ Institut \\ für Neurowissenschaften und Medizin (INM-1), Forschungszentrum Jülich, Jülich, Germany, ${ }^{4}$ Neurologische Klinik, Universität Marburg, \\ Marburg, Germany and ${ }^{5}$ Praxis für Humangenetik, Dresden, Germany \\ Email: Veronica Bernard - Veronica.Bernard@uk-sh.de; Martina Minnerop - m.minnerop@fz-juelich.de; Katrin Bürk - buerk@ngi.de; \\ Friedmar Kreuz - f.kreuz@medizinische-genetik-dresden.de; Gabriele Gillessen-Kaesbach - g.gillessen@uk-sh.de; \\ Christine Zühlke* - christine.zuehlke@uk-sh.de \\ * Corresponding author
}

Published: II September 2009

BMC Medical Genetics 2009, 10:87 doi:10.1186/1471-2350-10-87
Received: 17 April 2009

Accepted: II September 2009

This article is available from: http://www.biomedcentral.com/I47/-2350/10/87

(c) 2009 Bernard et al; licensee BioMed Central Ltd.

This is an Open Access article distributed under the terms of the Creative Commons Attribution License (http://creativecommons.org/licenses/by/2.0), which permits unrestricted use, distribution, and reproduction in any medium, provided the original work is properly cited.

\begin{abstract}
Background: The autosomal recessively inherited ataxia with oculomotor apraxia 2 (AOA2) is a neurodegenerative disorder characterized by juvenile or adolescent age of onset, gait ataxia, cerebellar atrophy, axonal sensorimotor neuropathy, oculomotor apraxia, and elevated serum AFP levels. AOA2 is caused by mutations within the senataxin gene (SETX). The majority of known mutations are nonsense, missense, and splice site mutations, as well as small deletions and insertions.
\end{abstract}

Methods: To detect mutations in patients showing a clinical phenotype consistent with AOA2, the coding region including splice sites of the SETX gene was sequenced and dosage analyses for all exons were performed on genomic DNA. The sequence of cDNA fragments of alternative transcripts isolated after RT-PCR was determined.

Results: Sequence analyses of the SETX gene in four patients revealed a heterozygous nonsense mutation or a 4 bp deletion in three cases. In another patient, PCR amplification of exon II to I5 dropped out. Dosage analyses and breakpoint localisation yielded a $1.3 \mathrm{~kb}$ LINEI insertion in exon $\mathrm{I} 2$ (patient $\mathrm{PI}$ ) and a $6.1 \mathrm{~kb}$ deletion between intron II and intron $\mathrm{I} 4$ (patient $\mathrm{P} 2$ ) in addition to the heterozygous nonsense mutation R 1606X. Patient P3 was compound heterozygous for a 4 bp deletion in exon 10 and a $20.7 \mathrm{~kb}$ deletion between intron 10 and 15. This deletion was present in a homozygous state in patient P4.

Conclusion: Our findings indicate that gross mutations seem to be a frequent cause of $A O A 2$ and reveal the importance of additional copy number analysis for routine diagnostics. 


\section{Background}

The autosomal recessive cerebellar ataxias (ARCA) represent a phenotypically and genetically heterogeneous group of neurodegenerative disorders mainly beginning before 20 years of age.

More than 20 different forms of ARCA have been described. Friedreich ataxia (FRDA) is the most frequent form in Europe followed by ataxia with oculomotor apraxia 2 (AOA2, OMIM \#606002) [1]. AOA2 is genetically defined by mutations in the senataxin gene (SETX) (OMIM *608465) located on chromosome 9q34. Patients typically present with early onset ataxia (range: 3-30 years), peripheral axonal sensorimotor neuropathy with areflexia ( $>90 \%$ of individuals), oculomotor apraxia ( $<50 \%$ of individuals), marked cerebellar atrophy on MRI, and slow progression [2]. Dystonic hand posture, choreic movements, and head or postural tremor are present in about $20 \%$ of individuals. Serum $\alpha$-fetoprotein (AFP) concentration is elevated in $>90 \%$ of affected individuals. In $50 \%$ of patients, serum cholesterol levels are increased. Serum creatine kinase (CK) may be abnormal in patients with severe amyotrophy.

Mutations in SETX were first reported in 2004 [2]. The SETX gene consisting of 26 exons (coding exons 3-26) encodes for senataxin, a 2677 amino acid protein containing a putative DNA/RNA helicase domain. This helicase domain possesses strong homology to yeast RNA helicase Sen $1 p$. To date, at least 51 mutations within the SETX gene responsible for the AOA2 phenotype are known [214]. The majority are nonsense, missense, and splice site mutations as well as small deletions and insertions. AOA2 is allelic to ALS4, one form of amyotrophic lateral sclerosis with juvenile onset and autosomal dominant inheritance $[6,15]$.

In addition to numerous mutations of single or few nucleotides, four cases with large gene rearrangements within SETX have been described in patients with AOA2. A 20.6 $\mathrm{kb}$ deletion (intron 15 to intron 23) was identified in an Italian family [7], a 10 kb duplication was found in a German patient [4] and in two families from Algeria a deletion of exon 7 and a deletion of exon 19 and 20 have been reported [14].

Samples of patients with gait instability, areflexia, neuropathy, cerebellar dysarthria, and oculomotor signs were screened for mutations in the SETX gene. In six patients, clinical diagnosis of AOA2 could be confirmed by sequence analyses [13]. Additionally, there was evidence for compound heterozygous deletions, insertions as well as homozygous deletions in AOA2 patients.

\section{Methods \\ Patients}

After obtaining informed consent, DNA and RNA were extracted from peripheral blood leukocytes using standard procedures. The study was approved by the Ethic Committee of the University to Lübeck (reference number: 09-041) in compliance with the Helsinki Declaration. Clinical data are summarized in Table 1.

Patient P1 showed first signs of gait imbalance at 12 years of age. At the age of 25 years, he presented with marked ataxia and used a stroller. He also had oculomotor signs including oculomotor apraxia, cerebellar dysarthria, neuropathy with muscular atrophy and areflexia of upper and lower limbs. Serum AFP was elevated (9.7 ng/ml).

Patient P2 noticed first gait problems when he was 15 years old. At the age of 28, he presented with marked ataxia of gait and stance using a stroller for longer distances. He also had oculomotor apraxia, cerebellar dysarthria, neuropathy with muscular atrophy and areflexia of upper and lower limbs. Serum AFP was elevated (12.6 ng/ $\mathrm{ml})$.

In patient P3, ataxia started at the age of 12 . Seventeen years later, he was not able to walk without support. Clinically, he showed neuropathy with amyotrophy including small hand muscles, pes cavus, and dysarthria. Serum AFP was clearly elevated $(56 \mathrm{ng} / \mathrm{ml})$.

Patient P4 experienced first gait disturbances at the age of 12. During the following years, she developed progressive atrophy of distal muscles in the lower limbs. At the age of 33, there was evidence of cerebellar ataxia and sensorimotor neuropathy. Muscular atrophy was generalized with involvement of hand and proximal hip muscles and a positive Trendelenburg's sign. Oculomotor testing revealed major fixation instability, downbeat and gaze evoked nystagmus, saccadic pursuit and bilateral sixth cranial nerve palsy. There was no oculomotor apraxia.

\section{Sequencing analysis}

We screened for SETX mutations by direct sequencing of all 24 coding exons and flanking intronic sequences. PCR products were amplified using standard protocols (primer sequences available on request). After ExoSAP-IT treatment (USB Inc, Staufen, Germany) of the PCR products, sequencing reactions were performed using the BigDye Terminator v1.1 Sequencing Standard Kit (Applied Biosystems Inc, Darmstadt, Germany) and analysed on the automated capillary sequencer $3130 x$ l Genetic Analyser (Applied Biosystems Inc, Darmstadt, Germany). 
Table I: Clinical data at last presentation. MRI showed global cerebellar atrophy.

\begin{tabular}{|c|c|c|c|c|c|c|c|c|c|c|c|c|c|}
\hline patient & age & $\operatorname{sex}$ & DD & $\begin{array}{c}\text { cerebellar } \\
\text { ataxia }\end{array}$ & $\begin{array}{l}\text { oculomoto } \\
\text { r apraxia }\end{array}$ & neuropathy & dystonia & $\begin{array}{l}\text { Pyramidal } \\
\text { signs }\end{array}$ & dementia & MRI & AFP & cholesterol & CK \\
\hline no. & [years] & & [years] & & & & & & (MMSE) & (atrophy) & {$[<5 \mathrm{ng} / \mathrm{ml}]$} & [<220 mg/dl] & {$[<|7|$ UII] } \\
\hline 1 & 25 & $\mathrm{~m}$ & 13 & yes & yes & yes & no & no & $\begin{array}{c}\text { No } \\
(27 / 30)\end{array}$ & cerebellum & 9.7 & 179 & 138 \\
\hline 2 & 28 & $\mathrm{~m}$ & 13 & yes & yes & yes & no & no & $\begin{array}{c}\text { No } \\
(30 / 30)\end{array}$ & cerebellum & 12.6 & normal & 193 \\
\hline 3 & 29 & $\mathrm{~m}$ & 17 & yes & yes & yes & no & no & no & cerebellum & 56 & 155 & 220 \\
\hline 4 & 33 & $f$ & 21 & yes & no & yes & no & no & $\begin{array}{c}\text { No } \\
(30 / 30)\end{array}$ & cerebellum & 32 & n.a. & 171 \\
\hline
\end{tabular}

$\mathrm{m} / \mathrm{f}=$ male/female

$\mathrm{DD}=$ disease duration

MMSE = Mini Mental State Examination (Folstein et al., J Psychiatr Res 1975; 1225.4: 189-198)

AFP = alpha-fetoprotein

CK $=$ creatin kinase

n.a. $=$ not available 


\section{Haplotype analyses}

In case of identical mutations in unrelated patients, haplotype analyses for the AOA2 region with the markers D9S159, D9S1831, D9S1863, D9S1847, D9S1830 and D9S1793 (NCBI database, primer sequences are listed in additional file 1) were performed. FAM-labelled PCR products were separated on automated capillary sequencer 3100-Avant Genetic Analyser (Applied Biosystems Inc, Darmstadt, Germany).

\section{Breakpoint localisation}

Dosage analyses were performed on genomic DNA for the 24 coding exons of the SETX gene using the ABI 7300 Real Time PCR System (Applied Biosystems Inc, Darmstadt, Germany) in the presence of SYBR-Green (SYBR-Green I core reagent kit including AmpliTaq-GOLD polymerase, Applied Biosystems Inc, Darmstadt, Germany). The optimisation of the PCR reaction was performed according to the manufacturer's instructions (Applied Biosystems Inc, Darmstadt, Germany, User Bulletin 2 applied to the SYBRGreen I core reagent protocol) but scaled down to $25 \mu \mathrm{l}$ per reaction.

Long-range PCR was performed using the Expand High Fidelity PCR System (Roche Diagnostics GmbH, Mannheim, Germany). Primers flanking the potential deletion were used. Whenever the amplification at genomic DNA level failed, the regions of possible deletion breakpoints were narrowed down by primer walking. Subsequently, PCR on genomic DNA across the deletion junctions were done using standard protocols (Primer sequences for breakpoint localisation are listed in additional file 2). PCR products have been verified by sequencing analysis.

\section{RNA analysis}

For RNA analyses, total RNA was isolated from peripheral blood leukocytes using PAXgene kit according to the manual (PreAnalytiX, Hombrechtikon, Switzerland). Reverse transcriptase PCR was performed with the OneStep RTPCR Kit (Qiagen, Hilden, Germany) according to manufacturer's recommendations. For RT-PCR, gene specific primers flanking the presumptive mutation were used (primers sequences are listed in additional file 3). Amplified products were separated on $0.8 \%$ agarose gels. Separated fragments were excised from the gel and eluted using the Perfectprep Gel Cleanup kit (Eppendorf, Hamburg, Germany). Extracted DNA was sequenced as mentioned above.

\section{Sequence Analysis}

Sequence analyses were performed using the GenBank reference sequence (Accession number: NM 015046). Repetitive elements were analysed using RepeatMasker version open-3.2.7 http://www.repeatmasker.org.

\section{Results \\ Detection of small mutations in the SETX gene by sequencing analysis}

Sequencing the coding region of the SETX gene revealed heterozygous mutations in three patients (Table 2). In patient P4, PCR amplification of exon 11 to 15 failed.

Patient P1 and P2 were heterozygous for a C>T transition at position 4816 of the cDNA, that encodes a stop codon (c.4816C>T, p.R1606X). In both cases, heterozygosity for this nonsense mutation was also present in the maternal DNA. Haplotype analyses excluded a common founder for this nonsense mutation.

Table 2: Mutations in SETX

\begin{tabular}{|c|c|c|c|}
\hline DNA & Alteration in DNA (Exon) & Alteration in Protein & Mutation Status \\
\hline \multirow[t]{2}{*}{ Patient I } & c. $4816 \mathrm{C}>\mathrm{T}(10)$ & p.RI606X & $\begin{array}{l}\text { compound } \\
\text { heterozygous }\end{array}$ \\
\hline & c.540I_5402ins I 280bp & p.VI792_LI8I3del, p.VI792_MI850delinsV & \\
\hline \multirow[t]{2}{*}{ Patient 2} & c. $4816 \mathrm{C}>\mathrm{T}(10)$ & p.RI606X & $\begin{array}{l}\text { compound } \\
\text { heterozygous }\end{array}$ \\
\hline & c.5374+9369_5950-254del6107bp & p.VI792EfsX3I, p.VI792_L2035del & \\
\hline \multirow[t]{2}{*}{ Patient 3} & c.4633_4636delAGTG & p.SI545AfsX26 & $\begin{array}{l}\text { compound } \\
\text { heterozygous }\end{array}$ \\
\hline & $\begin{array}{l}\text { c.5274+13396_6107-3547 } \\
\text { del20729bp }\end{array}$ & p.VI759Efs X6 & \\
\hline Patient 4 & $\begin{array}{l}\text { c.5274+13396_6107-3547 } \\
\text { del20729bp }\end{array}$ & p.VI759Efs X6 & homozygous \\
\hline
\end{tabular}




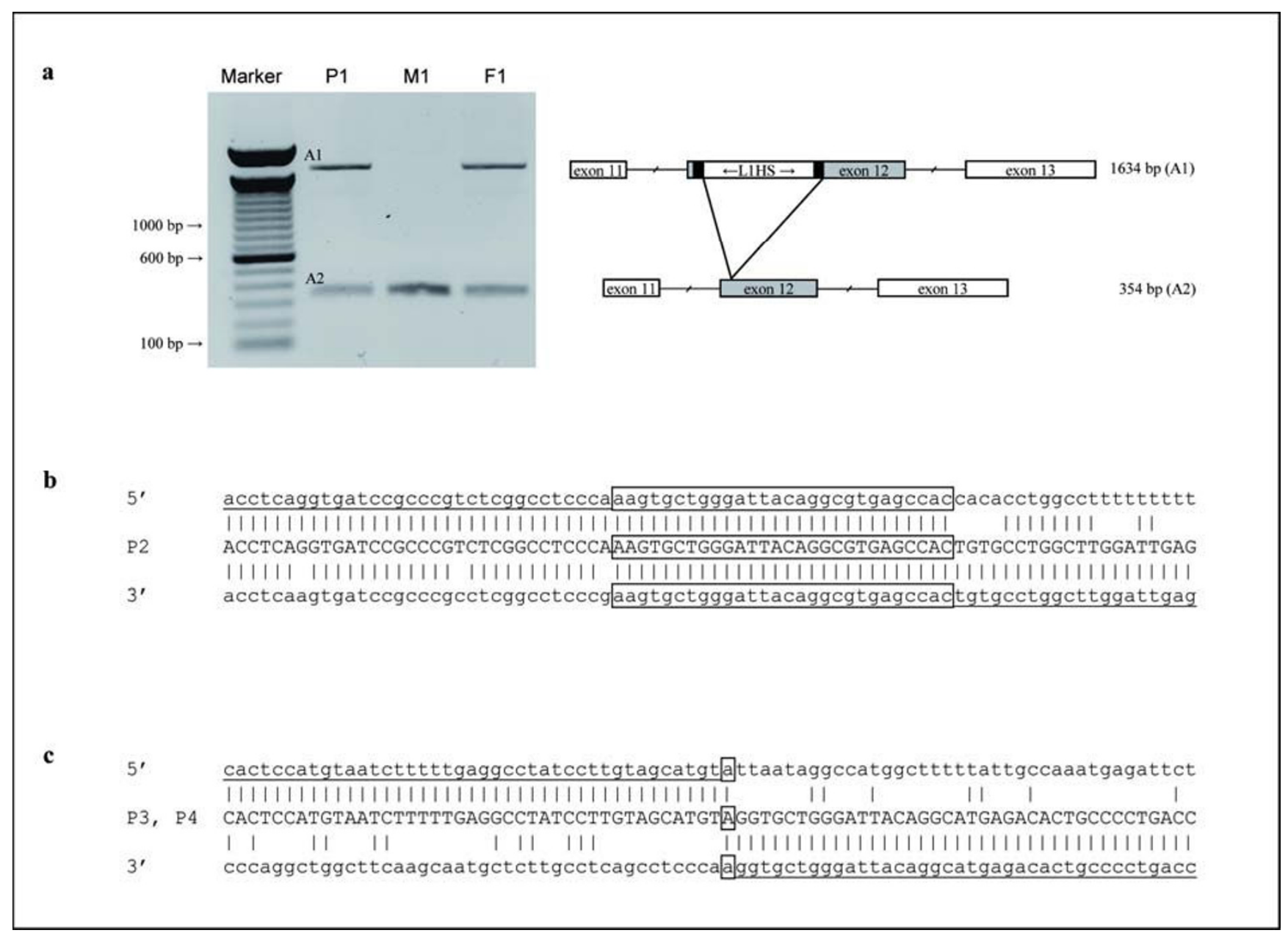

\section{Figure I}

Localisation of the Insertion and Deletion Breakpoints. (a) Long range PCR products for patient PI, his mother MI, and his father $\mathrm{FI}$ separated on a $0.8 \%$ agarose gel. Marker: 100 bp DNA Ladder. Amplicon A2 represents the wildtype fragment and AI the PCR product with the LIHS insertion. Schematic drawing shows the LIHS insertion in exon I2. Exons are indicated as boxes, introns as interrupted lines. The LIHS insertion is flanked by a 15 bp target site duplication (black boxes). (b) Sequence of the breakpoint junction in patient P2 compared to control sequence. Homologous regions are boxed. (c) Sequence alignment of the breakpoint junction in patients P3 and P4 and the control 5' and 3' regions.

Patient P3 was heterozygous for a 4 bp deletion in exon 10 of the SETX gene (c.4633_4636delAGTG). This deletion leads to a frame shift and generates a premature stop codon (p.S1545AfsX26). Heterozygosity for this mutation was also confirmed in the mother.

\section{Searching for gross changes in the SETX gene by dosage analysis}

The presence of heterozygous SETX mutations in three patients presenting with a clinical phenotype consistent with AOA2 suggested the implementation of dosage analyses to detect potential copy number variations. Reproducible aberrant signals were found in patients P1, P2, and P3 (data not shown). Patient P1 showed a 50\% decrease for exon 12 compared to different exons of the gene. Patient P2 had reduced values for exon 12 to 14 . In patient P3, a shifting to decreased gene dosage was detected for exon 11 to 15 .

\section{Identification of mutations by long-range PCR and breakpoint analysis}

In patient P1, long-range PCR on genomic DNA using primers flanking exon 12 revealed the 354 bp wildtype fragment and an additional $~ 1.6 \mathrm{~kb}$ PCR product (P1, Figure 1a). The same pattern could be observed in the paternal DNA (F1), whereas the mother (M1) showed the wildtype fragment. Sequencing of the $1.6 \mathrm{~kb}$ PCR product indicated a $1.3 \mathrm{~kb}$ insertion within exon 12 (c.5401_5402ins1280bp). The insertion consists of a $5^{\prime}$ truncated L1HS element. The first part of the 5 ' truncated 


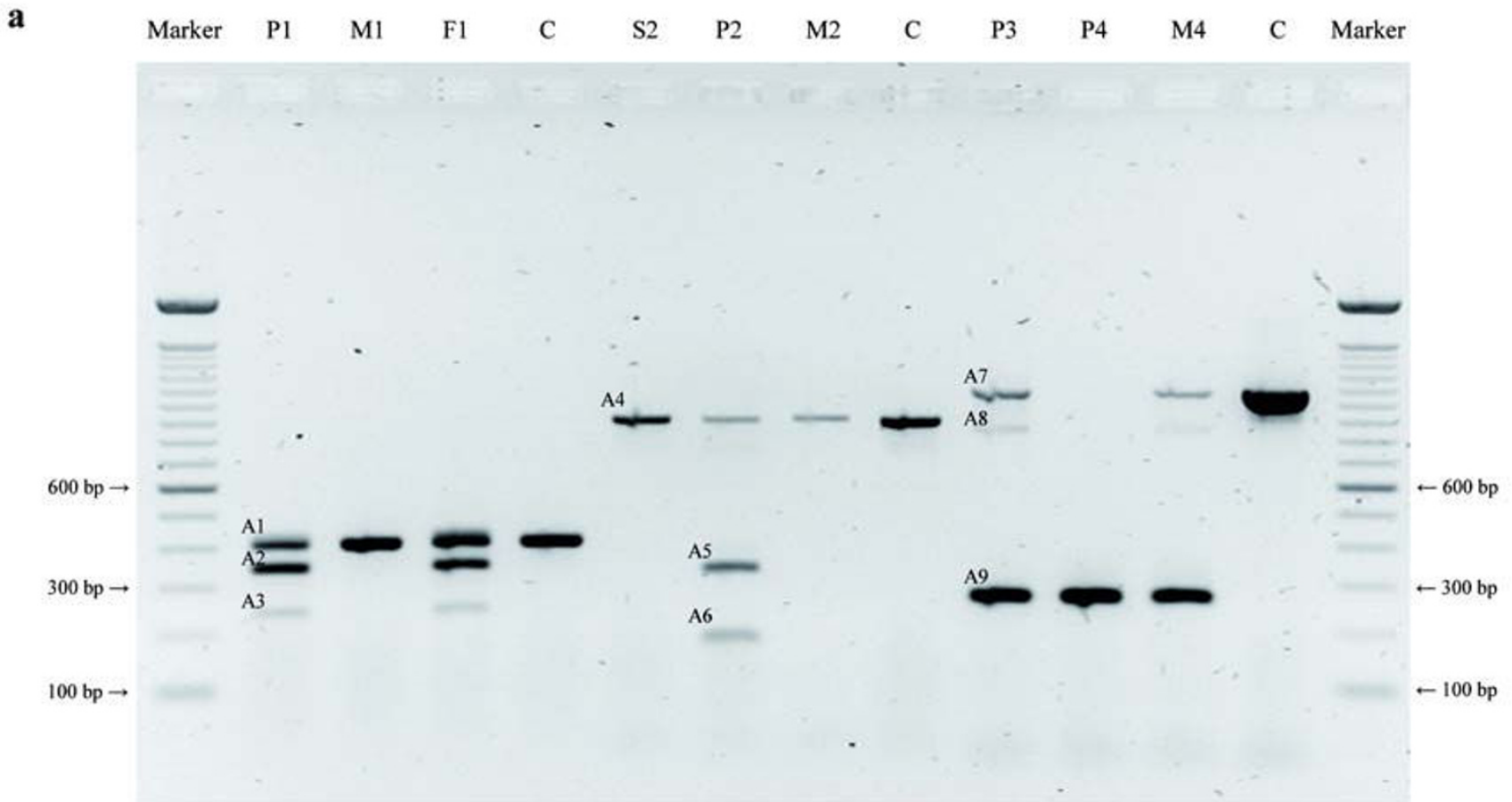

b

\begin{tabular}{|c|c|c|c|}
\hline exon 11 & exon 12 & exon 13 & \multirow[t]{2}{*}{$416 \mathrm{bp}(\mathrm{A} 1)$} \\
\hline & \multicolumn{2}{|c|}{$r$} & \\
\hline & \begin{tabular}{|l|} 
exon 12 \\
\end{tabular} & exon 13 & \multirow[t]{2}{*}{$350 \mathrm{bp}(\mathrm{A} 2)$} \\
\hline & $\underbrace{r}$ & & \\
\hline
\end{tabular}

P2

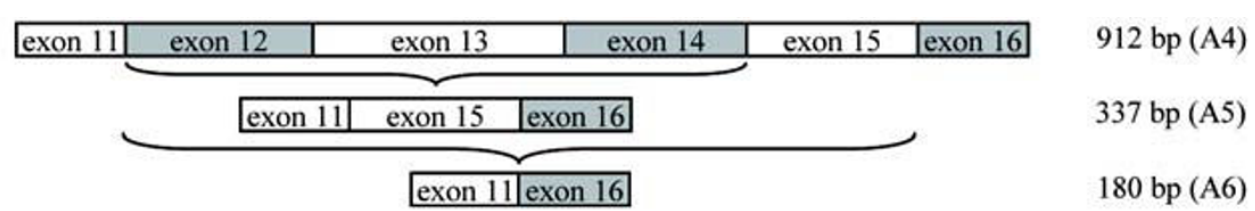

\section{P3, P4}

\begin{tabular}{|c|c|c|c|c|c|c|}
\hline exon 10 (end) & exon 11 & exon 12 & exon 13 & exon 14 & exon 15 & exon 16 \\
\hline
\end{tabular}

$1106 \mathrm{bp}(\mathrm{A} 7)$

274 bp (A9)

Figure 2

Aberrant Transcripts identified by RT-PCR. (a) RT-PCR products separated on a $0.8 \%$ agarose gel. Marker: 100 bp DNA Ladder, AI-A9: PCR products, M: mother, F: father, S: sister, C: control. (b) Schematic maps of the RT-PCR. Exons (boxes) are represented to scale. 
L1HS element is orientated in antisense with respect to the disrupted gene, whereas the second part is directed in sense. The insert is flanked by a 15 bp duplicated region. This insertion detected by long-range PCR escaped routine sequencing due to its size.

For patients P2, P3, and P4, breakpoints were narrowed down by primer walking. Patient P2 showed a decreased dosage for intron 11 and 14. Primers flanking the predicted deletion resulted in a PCR product spanning the deletion breakpoints. Sequence analysis revealed a $6.1 \mathrm{~kb}$ deletion between intron 11 and 14 (c.5374+9369_5950254del6107bp). This deletion event occured within Alu elements. Figure $1 \mathrm{~b}$ depicts a sequence alignment of the identified breakpoint junction in patient P2 and the wildtype 5' and 3' regions. The sequences are characterised by high degree of homology and the junction contains 28 bp of microhomology (boxed, Figure 1b).

In patient P3, altered gene dosages were observed within introns 10 and 15. Sequencing of PCR products spanning the deletion breakpoints revealed a $20.7 \mathrm{~kb}$ deletion (c.5274+13396_6107-3547del20729bp). The 5' breakpoint is located within a LINE1 element in intron 10 and the 3' breakpoint is placed between an AluY and a L1ME3 element in intron 15. Patient P4 was homozygous for this deletion. Sequences surrounding the deletion breakpoints in comparison to the junctions in patient P3 and patient P4 are depicted in Figure 1c. There was no evidence for sequence homology of the breakpoints.

Haplotype analyses of the complete $5.4 \mathrm{cM}$ interval between D9S159 and D9S1793 did not show a common genotype for patient P3 and P4 (additional file 4). However, both patients shared a common allele for chromosome 9 markers D9S1847 and D9S1830 that are located next to the SETX gene.

\section{Expression of mutated SETX alleles}

In leukocytes of all four patients, aberrant transcripts could be identified by RT-PCR (Figure 2). Amplification of cDNA from exon 11 to exon 13 revealed two additional products (A2 and A3) in patient P1 and his father F1. The mother $\mathrm{M} 1$ and the control $\mathrm{C}$ carried the expected wildtype fragment (A1, Figure 2a). In addition to this wildtype fragment (416 bp, A1), sequence analysis yielded a transcript lacking the first 66 bp of exon 12 (350 $\mathrm{bp}, \mathrm{A} 2)$ and another transcript missing the complete exon 12 (242 bp, A3).

In patient P2, three fragments could be detected by RTPCR using exonic primers from exons 11 and 16. In addition to the wildtype fragment A4 (912 bp) that was present in the cDNA of the healthy sister $\mathrm{S} 2$, the mother $\mathrm{M} 2$, and in the control sample, two shortened products lacking exons 12 to 14 (337 bp, A5) or exons 12 to 15 (180 bp, A6) could be found.

For patient P3, two products resulting from RT-PCR with primers amplifying exons 10 to 16 were subjected to further investigations: The upper $1106 \mathrm{bp}$ fragment A7 represented the wildtype sequence. In the lower 274 bp fragment (A9), exon 10 was found to be spliced to exon 16.

Sequencing yielded an additional faint signal (A8) corresponding to a heteroduplex composed of A7 and A9. In the cDNA of patient P4, the 274 bp fragment (A9) was observed in the absence of the wildtype transcript. The mother M4 of patient P4 being heterozygous for the mutation, displayed the same expression pattern as patient P3, while the control sample exclusively showed the wildtype fragment.

\section{Discussion}

In this study, we describe four patients with large mutations within the SETX gene. We have identified one insertion and two different deletions. Interestingly, all insertion and deletion events occurred within or near transposable elements (TE). Transposable elements are frequently recurring sequences spread all over the human genome. They comprise about $45 \%$ of the total genome sequence and can be categorized into four classes: short interspersed elements (SINE), long interspersed elements (LINE), LTR retrotransposons and DNA transposons [16].

The complex insertion in patient P1 showed homology to L1HS elements. L1HS elements are human specific LINE1 (L1) elements. Some full-length L1 elements are still capable of active retrotransposition. Retrotranspositionally competent L1 elements have reverse transcriptase and endonuclease activity [17]. L1-mediated insertions typically integrate at an endonuclease consensus cleavage site (3'-A/TTTT-5') and show characteristic hallmarks like 5' truncation, target site duplication and a long 3 ' poly A tail [18]. The $1.3 \mathrm{~kb}$ insertion detected in patient P1 has typical characteristics of L1-mediated retrotransposition. The retrotranspositional event occurred at the L1 endonuclease cleavage site $3^{\prime}-\mathrm{A} / \mathrm{TTTT}-5^{\prime}$. The insert harbours a $5^{\prime}$ truncation and a poly A tail. The entire element is flanked by a 15 bp target site duplication. L1 elements are thought to be inserted either in antisense or in sense orientation with respect to disrupted target genes [18]. However, in patient P1 the first part of the 5' truncated L1HS element was found to be orientated in antisense whereas the second part was directed in sense. This fact raises the question, if the insertion resulted from a single retrotranspositional event, or if the generation of this complex insertion is due to several independent steps. Irrespective of the generation of the insertion, this muta- 
tion seems to be stably inherited: The identical variation was also present in the father. Expression analyses revealed two aberrant splice variants for patient P1. Transcripts missing the first 66 bp of exon 12 as well as transcripts with loss of the complete exon 12 were detected. Both deletions are in-frame, therefore maintaining the open reading frame. The insertion seems to destroy the acceptor splice site of intron 11. In the case of the $66 \mathrm{bp}$ deletion an alternative splice site within exon 12 is used.

The deletion breakpoints for the $6.1 \mathrm{~kb}$ deletion in patient $\mathrm{P} 2$ and the $20.7 \mathrm{~kb}$ deletion in patients P3 and P4 are located in or near transposable elements. The $6.1 \mathrm{~kb}$ deletion occurred between two Alu elements, whereas the 20.7 $\mathrm{kb}$ deletion involved LINE1 and Alu elements. Interestingly, the known $\sim 10 \mathrm{~kb}$ duplication and the $\sim 20.6 \mathrm{~kb}$ deletion [4,7] also occurred within or near Alu elements.

Alu elements represent the major subgroup of SINE elements. RT-PCR with primers flanking the $6.1 \mathrm{~kb}$ deletion showed two aberrant transcripts for patient P2. In one transcript exons 12 to 14 were deleted while exons 12 to 15 were missing in another transcript. The $6.1 \mathrm{~kb}$ deletion occurred between introns 11 and 14. The acceptor splice site of intron 14 still exists at genomic level and seems to be partly functional. RNA analyses of the $20.7 \mathrm{~kb}$ deletion in patients $\mathrm{P} 3$ and $\mathrm{P} 4$ revealed an additional transcript lacking exons 11 to 15 . This result is consistent with the deletion spanning intron 10 to intron 15 at genomic level. Haplotype analyses may point to an ancient founder effect for the deletion in patient P3 and P4 originating from distinct parts of Germany due to the fact that the alleles for D9S1847 and D9S1830 are identical in both patients. Nevertheless, it should be underlined that these alleles are common in the German population (data not shown).

In all cases presented here, the gross mutations were found to be associated with transposable elements (TE). Interestingly, the $\sim 10 \mathrm{~kb}$ duplication and the $\sim 20.6 \mathrm{~kb}$ deletion reported before $[4,7]$ occurred within or near Alu elements, too. Human genes bearing a TE content $>40 \%$ seem to have an increased frequency of gross deletions [16]. Furthermore, the deletion breakpoints are predominantly located in TE subclasses that are specifically overrepresented in the involved gene compared to the human genome [16]. Repetitive elements - mainly LINE1 and Alu elements - account for $47.5 \%$ of the entire SETX gene explaining the high number of deletions and insertions.

Only four of 51 SETX mutations identified so far have been reported to be gross mutations $(7.8 \%)[4,7,14]$. In our own series, three of 15 distinct SETX mutations (20\%) were found to be large alterations. These discrepancies may result from methodological problems since gross mutations potentially escape routine diagnostics due to their size.

\section{Conclusion}

Gross mutations potentially may escape routine diagnostic due to their size. Thus, large deletions, insertions, and duplications are probably an underestimated cause for AOA2.

\section{Competing interests}

The authors declare that they have no competing interests.

\section{Authors' contributions}

$\mathrm{CZ}$ designed the study. VB carried out the molecular genetic work. MM, KB and FK investigated the patients and collected the clinical data. GG participated in the design of the study and helped to draft the manuscript. All authors read and approved the final manuscript.

\section{Additional material}

\author{
Additional file 1 \\ Primer sequences for chromosome 9 markers. This file contains the \\ primer sequences used for haplotype analyses. \\ Click here for file \\ [http://www.biomedcentral.com/content/supplementary/1471- \\ 2350-10-87-S1.doc]
}

\section{Additional file 2}

Primer sequences for breakpoint localisation. This file contains the primer sequences used for breakpoint localisation.

Click here for file

[http://www.biomedcentral.com/content/supplementary/14712350-10-87-S2.doc]

\section{Additional file 3}

Primer sequences for RT-PCR. This file contains the primer sequences used for RT-PCR.

Click here for file

[http://www.biomedcentral.com/content/supplementary/14712350-10-87-S3.doc]

\section{Additional file 4}

Linkage analysis for patient $P 3$ and patient P4. This file shows the linkage analysis for patient $P 3$ and patient P4. Chromosome 9 haplotypes between markers D9S159 and D9S1793 are shown.

Click here for file

[http://www.biomedcentral.com/content/supplementary/14712350-10-87-S4.ppt]

\section{Acknowledgements}

The authors thank all patients for providing blood samples for scientific research. Furthermore, they thank U. Gehlken for excellent technical assistance and Dr. A. Dalski for discussion. Part of this work was supported by the Deutsche Forschungsgemeinschaft (DFG: ZU 136/I-2) and by the German Heredo-Ataxia Society (DHAG). The authors declare that no conflicts of interest exist. 


\section{References}

I. Zühlke C, Bernard V, Gillessen-Kaesbach G: Investigation of recessive ataxia loci in patients with young age of onset. Neuropediatrics 2007, 38:207-209.

2. Moreira MC, Klur S, Watanabe M, Németh AH, Le Ber I, Moniz JC, Tranchant C, Aubourg P, Tazir M, Schöls L, Pandolfo M, Schulz JB, Pouget J, Calvas P, Shizuka-lkeda M, Shoji M, Tanaka M, Izatt L, Shaw CE, M'Zahem A, Dunne E, Bomont P, Benhassine T, Bouslam N, Stevanin $G$, Brice A, Guimarães J, Mendonça $P$, Barbot $C$, Coutinho $P$, Sequeiros J, Dürr A, Warter JM, Koenig M: Senataxin, the ortholog of a yeast RNA helicase, is mutant in ataxia-ocular apraxia 2. Nat Genet 2004, 36:225-227.

3. Anheim M, Fleury MC, Franques J, Moreira MC, Delaunoy JP, StoppaLyonnet D, Koenig M, Tranchant C: Clinical and molecular findings of ataxia with oculomotor apraxia type 2 in 4 families. Arch Neurol 2008, 65:958-962.

4. Arning L, Schöls L, Cin H, Souquet M, Epplen JT, Timmann D: Identification and characterisation of a large senataxin (SETX) gene duplication in ataxia with ocular apraxia type 2 (AOA2). Neurogenetics 2008, 9:295-299.

5. Asaka T, Yokoji H, Ito J, Yamaguchi K, Matsushima A: Autosomal recessive ataxia with peripheral neuropathy and elevated AFP: Novel mutations in SETX. Neurology 2006, 66:1580-158I.

6. Chen YZ, Hashemi SH, Anderson SK, Huang Y, Moreira MC, Lynch DR, Glass IA, Chance PF, Bennett CL: Senataxin, the yeast Sen I p orthologue: Characterization of a unique protein in which recessive mutations cause ataxia and dominant mutations cause motor neuron disease. Neurobiology of Disease 2006, 23:97-108

7. Criscuolo C, Chessa L, Di Giandomenico S, Mancini P, Saccà F, Grieco GS, Piane M, Barbieri F, De Michele G, Banfi S, Pierelli F, Rizzuto N, Santorelli FM, Gallosti L, Filla A, Casali C: Ataxia with oculomotor apraxia type 2. A clinical, pathologic, and genetic study. Neurology 2006, 66: I 207-1210.

8. Duquette A, Roddier K, McNabb-Baltar J, Gosselin I, St-Denis A, Dicaire MJ, Loisel L, Labuda D, Marchand L, Mathieu J, Bouchard JP, Brais $B$ : Mutations in senataxin responsible for Quebec cluster of ataxia with neuropathy. Ann Neurol 2005, 57:408-4I4.

9. Fogel BL, Perlman S: Novel mutations in the senataxin DNA/ RNA helicase domain in ataxia with oculomotor apraxia 2. Neurology 2006, 67:2083-2084.

10. Lynch DR, Braastad CD, Nagan N: Ovarian failure in ataxia with oculomotor apraxia type 2. Am J Med Genet 2007, | 43A: I775-I777.

II. Nicolaou P, Georghiou, Votsi C, Middleton LT, Zamba-Papanicolaou E, Christodoulou K: A novel c.5308 53 I IdelGAGA mutation in senataxin in a Cypriot family with an autosomal recessive cerebellar ataxia. BMC Medical Genetics 2008, 9:28.

12. Schöls L, Arning L, Schüle R, Epplen JT, Timmann D: "Pseudodominant inheritance" of ataxia with ocular apraxia type 2 (AOA2). J Neurol 2008, 255:495-501.

13. Bernard V, Stricker S, Kreuz F, Minnerop M, Gillessen-Kaesbach G Zühlke C: Ataxia with oculomotor apraxia type 2: Novel mutations in six patients with juvenile age of onset and elevated serum alpha-fetoprotein. Neuropediatrics 2008 , 39:347-350.

14. Tazir M, Ali-Pacha L, M'Zahem A, Delaunoy JP, Fritsch M, Nouioua S, Benhassine T, Assami S, Grid D, Vallat JM, Hamri A, Koenig M: Ataxia with oculomotor apraxia type 2: A clinical and genetic study of 19 patients. Journal of the Neurological Sciences 2009, 278:77-81.

15. Chen YZ, Bennett CL, Huynh HM, Blair IP, Puls I, Irobi J, Dierick I, Abel A, Kennerson ML, Rabin BA, Nicholson GA, Auer-Grumbach M, Wagner K, De Jonghe P, Griffin JW, Fischbeck KH, Timmerman V, Cornblath DR, Chance PF: DNA/RNA helicase gene mutations in a form of juvenile amyotrophic lateral sclerosis (ALS4). Am J Hum Genet 2004, 74: I I 28- I I 35.

16. van Zelm MC, Geertsema C, Nieuwenhuis N, de Ridder D, Conley ME, Schiff C, Tezcan I, Bernatowska E, Hartwig NG, Sanders EA, Litzman J, Kondratenko I, van Dongen JJ, Burg M van der: Gross deletions involving IGHM, BTK, or Artemis: A model for Genomic Lesions Mediated by Transposable elements. Am J Hum Genet 2008, 82:320-332.

17. Kazazian $\mathrm{HH}$ Jr: Mobile elements: drivers of genome evolution. Science 2004, 303:1626-1632.
18. Chen JM, Stenson PD, Cooper DN, Férec C: A systematic analysis of LINE-I endonuclease-dependent retrotranspositional events causing human genetic disease. Human Genet 2005, | | 7:4 | |-427.

\section{Pre-publication history}

The pre-publication history for this paper can be accessed here:

http://www.biomedcentral.com/1471-2350/10/87/pre pub
Publish with Biomed Central and every scientist can read your work free of charge

"BioMed Central will be the most significant development for disseminating the results of biomedical research in our lifetime. "

Sir Paul Nurse, Cancer Research UK

Your research papers will be:

- available free of charge to the entire biomedical community

- peer reviewed and published immediately upon acceptance

- cited in PubMed and archived on PubMed Central

- yours - you keep the copyright 